\title{
Communication
}

\section{Elevated levels of serum antibodies against alpha-1, 6-glucan in patients with systemic lupus erythematosus or rheumatoid arthritis}

\author{
Hui Dai ${ }^{1}$, Xiao-Ming Gao ${ }^{2 凶}$ \\ ${ }^{1}$ Department of Immunology, Peking University Health Science Center, Peking University, Beijing 100083, China \\ 2 Institute of Biology and Medical Sciences, Soochow University, Suzhou 215123, China \\ \ Correspondence: xmgao@suda.edu.cn \\ Received July 19, 2011 Accepted September 1, 2011
}

\section{ABSTRACT}

This study was undertaken to investigate whether levels of anti-alpha-1, 6-glucan antibodies in human sera correlate with rheumatoid arthritis (RA) and systemic lupus erythematosus (SLE). Serum samples were collected from patients with SLE $(n=30)$, RA $(n=30)$ and healthy adult volunteers. IgG, IgA and IgM levels against alpha-1, 6-glucan were measured using enzyme linked immunosorbent assays. Anti-alpha-1, 6-glucan IgG prevalence was raised in patients with active SLE $(73.3 \%)$ and RA $(60 \%)$ compared with healthy controls $(13.3 \%)$. Strong correlation between anti-alpha-1,6-glucan-lgG levels and anti-perinuclear factor $(r=0.642 ; p<0.05)$ in RA patients or anti-nuclear antibodies $(r=0.675 ; p<0.05)$ in SLE patients was observed. No significant differences in anti-alpha-1,6-glucan-IgA or-IgM levels were noted between different groups. We conclude that anti-alpha1,6-glucan-IgG levels were significantly elevated in patients with SLE or RA and positively correlated with disease activity.

KEYWORDS autoantibody, anti-alpha-1, 6-glucan, autoimmune diseases, systemic lupus erythematosus, rheumatoid arthritis

\section{INTRODUCTION}

Humans have circulating natural antibodies towards a variety of glycans that exist on the surface of bacteria, fungi and parasites or on self-tissue cells, such as erythrocytes and endothelial cells. Such natural antibodies may play important roles in host defense against microbial invasion and/or in immunoregulation. Amongst the well-studied anti-glycan natural antibodies in humans are the anti-blood group antibodies (Pal et al., 2004; Dinglasan et al., 2005) and antiGal-a-1,3-Gal antibodies (Galili, 2005; Huang et al., 2006). Other known anti-carbohydrate human natural antibodies include those specific for Gal- $\beta-1,3-G a l N a c$ (T antigen) and lactose (Gupta et al., 1996; Galili, 2001). Some glycanspecific antibodies could be serological markers for autoimmune diseases. Dextran and anti-dextran antibodies have been known to appear in patients with gastrointestinal diseases and some colorectal carcinomas. These disorders seem to be linked to gut pathology, which is underlined by the facts that arthritis is an extraintestinal manifestation of Crohn's disease and that rheumatic diseases occur in patients with gluten-enteropathy more frequently than in the general population (Greenstein et al., 1976; Bourne et al., 1985). However, there is no hard evidence suggesting that dextran epitopes are specifically present in the gut. It is thus possible that a higher prevalence of anti-dextran antibodies might also be seen in patients with systemic rheumatic diseases, such as rheumatoid arthritis (RA) or systemic lupus erythematosus (SLE), which could occur irrespective of gut involvement. Our previous work showed that the prevalence of anti-Saccharomyces cerevisiae mannan antibodies (ASCMA) is elevated in both SLE and RA patients and levels of ASCMA are associated with disease activity in these patients (Dai et al., 2009a, b). We herein expand this study further analyzing whether levels of antibodies against dextran, a linear D-glucose polymer with $\alpha-1,6$-linkage, are also elevated in patients with SLE or RA. Results arising from this study may help us to better understand the pathogenesis of SLE or RA and also have important diagnostic and/or predictive values. 


\section{RESULTS}

\section{Elevated anti- $\alpha-1,6$-glucan-IgG level in SLE and RA}

Serum samples were screened for anti- $\alpha-1,6$-glucan lgG, IgM and IgA using dextran-based ELISA. The majority of samples from SLE patients $(73.3 \%)$ and RA patients $(60 \%)$ were positive for anti- $\alpha-1,6$-glucan lgG, significantly more than that observed in healthy subjects (13.3\%) (Fig. 1 and Table 1). Two of the high responder sera of SLE or RA patients were serially diluted respectively and assayed with an endpoint of $1: 800$ dilution, which confirmed the screening results (Fig. 2). We could not demonstrate that anti- $\alpha-1,6-$ glucan-IgM and-IgA levels in patients with SLE or RA were significantly higher than the control group (Table 1).

\section{Levels of anti- $\alpha-1,6-g$ lucan IgG correlate with disease activity}

Serum levels of $\alpha-1,6$-glucan-specific lgG in RA patients were compared with serological assay results including anti-keratin autoantibody (AKA), anti-perinuclear factor (APF), anti-cyclic citrullinated peptide (CCP), rheumatoid factor (RF), hidden rheumatoid factor for $\lg A / \lg G$ (HRFlgA/lgG), C-reactive protein (CRP) and ESR. As shown in Table 3, anti-a-1,6glucan-lgG levels strongly correlate with APF $(r=0.642$; $p<0.05)$.
Serum levels of $\alpha-1,6$-glucan-specific lgG in SLE patients were compared with that of anti-Smith antibodies (Sm), antihistone antibody (AHA), anti-RNP antibodies, antinuclear antibodies (ANA), anti-ribosomal $P$ protein antibody (ARPA), Ro (SSA) and La (SSB) antibodies. Interestingly, anti-a-1,6glucan-lgG levels strongly correlate with ANA in SLE ( $r=$ 0.675; $p<0.05$ ) (Table 2).

\section{ROC curve analysis of anti- $\alpha-1,6-$ glucan IgG}

ROC curves were plotted for anti- $\alpha-1,6$-glucan-IgG to evaluate sensitivity and specificity of our dextran-based ELISAs in diagnosing SLE or RA (Fig. 3). We also performed ROC curve analysis to determine the optimal cutoff for anti- $\alpha-1,6-$ glucan in SLE and RA. Healthy subjects were used as controls. For RA patients, with a cutoff value at 0.792 the sensitivity for detecting RA was $80 \%$, while the specificity was $80 \%$. 95\% confidence interval was between 0.6549-0.9073. The area under the ROC curve was 0.781 . For SLE patients, with a cutoff value at 1.09 the sensitivity for detecting SLE was $93.3 \%$, while the specificity was $73.3 \%$. 95\% confidence interval was between $0.7644-0.9623$.The area under the ROC curve was 0.863 .

\section{DISCUSSION}

Serum samples from patients with RA or SLE were compared
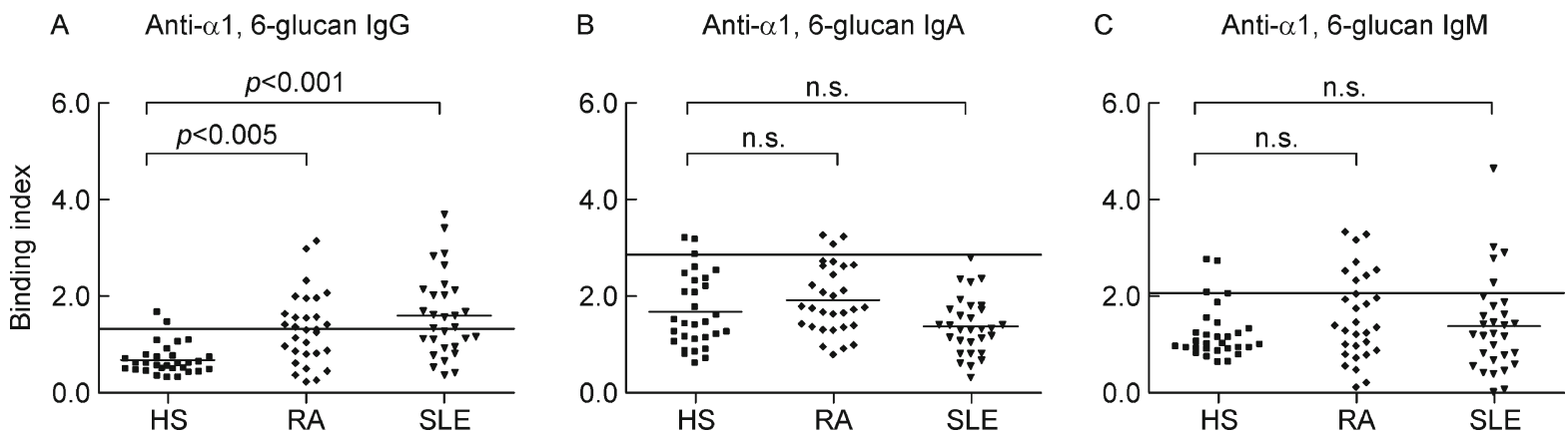

Figure 1. Comparison of anti- $\alpha-1,6-$ glucan-lgG, -lgA and-IgM levels in different groups. Serum samples from healthy adult human subjects (NS, $n=30)$, patients with SLE $(n=30)$, or RA $(n=30)$ were individually assayed for anti- $\alpha-1,6-$ glucan $\operatorname{lgG}(\mathrm{A})$, IgA (B) and IgM (C). The OD at $492 \mathrm{~nm}$ was measured and the reading for the negative control sera controlled at $0.2-0.28$. The results were calculated as binding indices and the mean binding indices of the 3 wells are shown. Horizontal lines represent cutoff values calculated using the ROC curves. Student's $t$ tests were performed comparing patient groups with the healthy controls and the $p$ values are given.

Table 1 Dextran-specific anbody prevalence in healthy subjects and patient groups ${ }^{a}$

\begin{tabular}{lccc}
\hline Anti-dextran autibodies & HS & RA & SLE \\
\hline $\lg G$ & $13.3 \%(4 / 30)$ & $60 \%(18 / 30), p=0.000^{\mathrm{b}}$ & $73.3 \%(22 / 30), p=0.000$ \\
$\lg \mathrm{l}$ & $23.3 \%(7 / 30)$ & $50 \%(15 / 30), p=0.073$ & $46.7 \%(14 / 30), p=0.461$ \\
$\lg$ & $36.7 \%(11 / 30)$ & $43.3 \%(13 / 30), p=0.184$ & $16.7 \%(5 / 30), p=0.108$ \\
\hline
\end{tabular}

${ }^{a}$ A summary of the data showing the prevalence (percent positive) of dextran-specific antibodies in healthy subjects (HS), patients with SLE or RA. The samples with binding indices above the cutoff values were scored as positive.

${ }^{\mathrm{b}}$ Student $t$ tests were performed comparing the patient groups with healthy adults and the $p$ values are shown. 

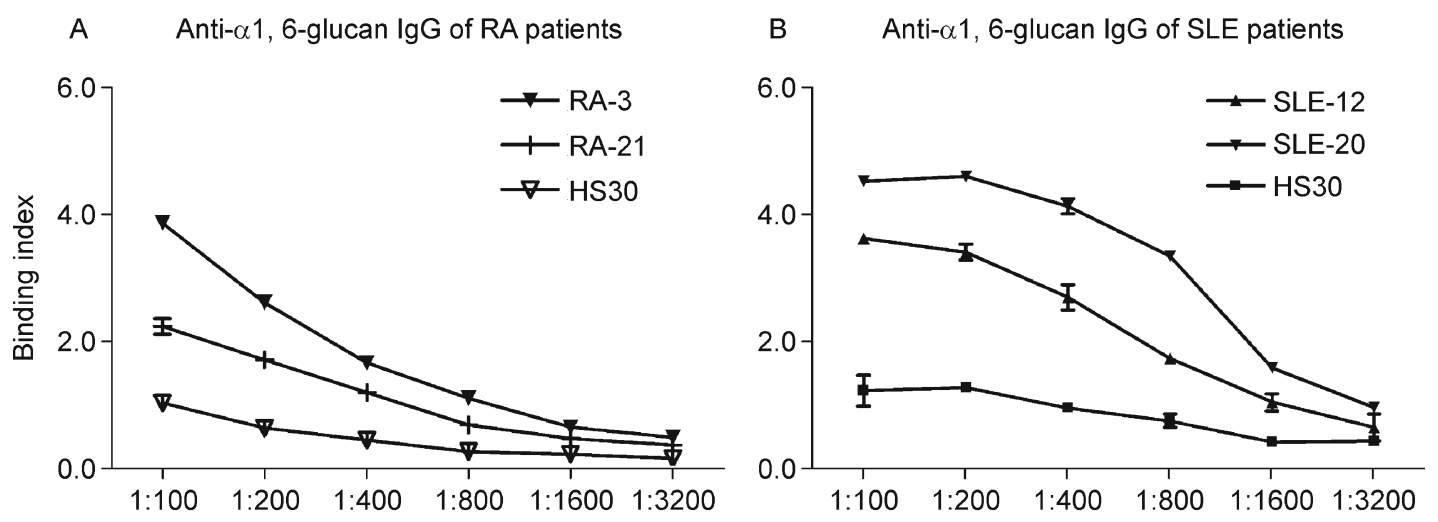

Serum dilution

Figure 2. Serum dilution assay. Serum samples from representative RA (A) and SLE (B) patients were serially diluted and assayed in parallel experiments to be compared with healthy subjects.

Table 2 Correlation analysis between anti- $\alpha-1,6-$ glucan antibodies and clinical index

\begin{tabular}{|c|c|c|c|c|}
\hline & $\begin{array}{c}\text { Clinical } \\
\text { index }\end{array}$ & $\begin{array}{l}\text { Correlation } \\
\text { coefficient }^{\mathrm{a}}\end{array}$ & $p$ (2-tailed $)^{b}$ & $n^{c}$ \\
\hline \multirow{8}{*}{ RA } & APF & 0.642 & $0.023^{*}$ & 29 \\
\hline & AKA & -0.258 & 0.193 & 28 \\
\hline & $\mathrm{CCP}$ & -0.182 & 0.373 & 28 \\
\hline & HRFIgA & 0.286 & 0.148 & 27 \\
\hline & HRFIgG & -0.067 & 0.739 & 27 \\
\hline & RF & -0.137 & 0.655 & 21 \\
\hline & CRP & 0.187 & 0.541 & 30 \\
\hline & ESR & 0.379 & 0.201 & 30 \\
\hline \multirow{7}{*}{ SLE } & ANA & 0.675 & $0.025^{*}$ & 28 \\
\hline & SSA & -0.118 & 0.536 & 30 \\
\hline & SSB & -0.266 & 0.163 & 29 \\
\hline & $\mathrm{Sm}$ & -0.114 & 0.556 & 29 \\
\hline & RNP & -0.125 & 0.518 & 29 \\
\hline & $\mathrm{AHA}$ & -0.238 & 0.57 & 12 \\
\hline & ARPA & -0.092 & 0.63 & 30 \\
\hline
\end{tabular}

with those from healthy subjects for antibodies against $\alpha-1,6-$ glucans using dextran-based ELISAs. The majority of patients with SLE $(73.3 \%)$ or RA $(60 \%)$ had elevated levels of serum IgG. Further studies of larger populations of various ethnic backgrounds are necessary to draw more definitive conclusions.

A key question is whether anti- $\alpha-1,6-$ glucan has any pathophysiological role in the pathogenesis of autoimmune diseases such as SLE and RA. It is unlikely, however, that antibodies against $\alpha$-glucans alone caused any pathological damage in vivo. The rabbit antibodies were able to compete with antibodies from patients with SLE and RA for binding with a-1,6-glucan, indicating that human and rabbit antibodies recognize the same, or at least spatially very close, epitope (data not shown), yet adult rabbit with high titer serum IgG against $\alpha-1,6$-glucan remained healthy (data not shown). Whether anti- $\alpha$-glucan antibodies, in combination with other factors such as a cytokine imbalance, play a part in the pathogenesis of autoimmune disorders in humans remains to be investigated. A prospective study on such individuals may evaluate whether this would have any predictive value for possible autoimmune disorders in their later lives.

Previous studies, using dextran-columns, have identified dextran-binding IgG and IgM in pooled plasma of healthy human subjects (Anastase et al., 1996; Chacko and Appukuttan, 2003). It should be emphasized that we assessed all serum samples at a 1:200 dilution, thus only capturing anti-glucan antibodies with a moderate to high titer. In contrast, the dextran-columns employed in previous investigations would detect low titer anti-dextran antibodies.

Dextran sulfate ligand could be bound to porous cellulose beads to form columns called selesorb. This kind of columns can adsorb pathogenic anti-DNA subgroups of high avidity and/or cationic antibodies, anticardiolipin, anti-CLbeta2GPI, and anaphylatoxins (Asahi et al., 2003). Patients with active SLE showed significantly higher titers of high-avidity antidsDNA antibody than did those with inactive SLE, and their titers were significantly reduced by immunoadsorption procedures (Hashimoto et al., 1991; Braun et al., 2002). The immunoadsorption therapy using dextran sulfate columns is effective in the removal of high-avidity anti-dsDNA antibodies that are closely associated with pathogenicity in SLE (Matsuki et al., 1996; Kutsuki et al., 1998). An open clinical study on 19 SLE cases revealed that the mean SLE disease activity index (SLEDAI) score significantly decreased from the pretreatment level of 10.2 to 4.5 after treatment (Suzuki, 2000). In our present work, significantly higher levels of anti-dextran IgG were detected in patients with SLE and the anti-dextran-IgG 

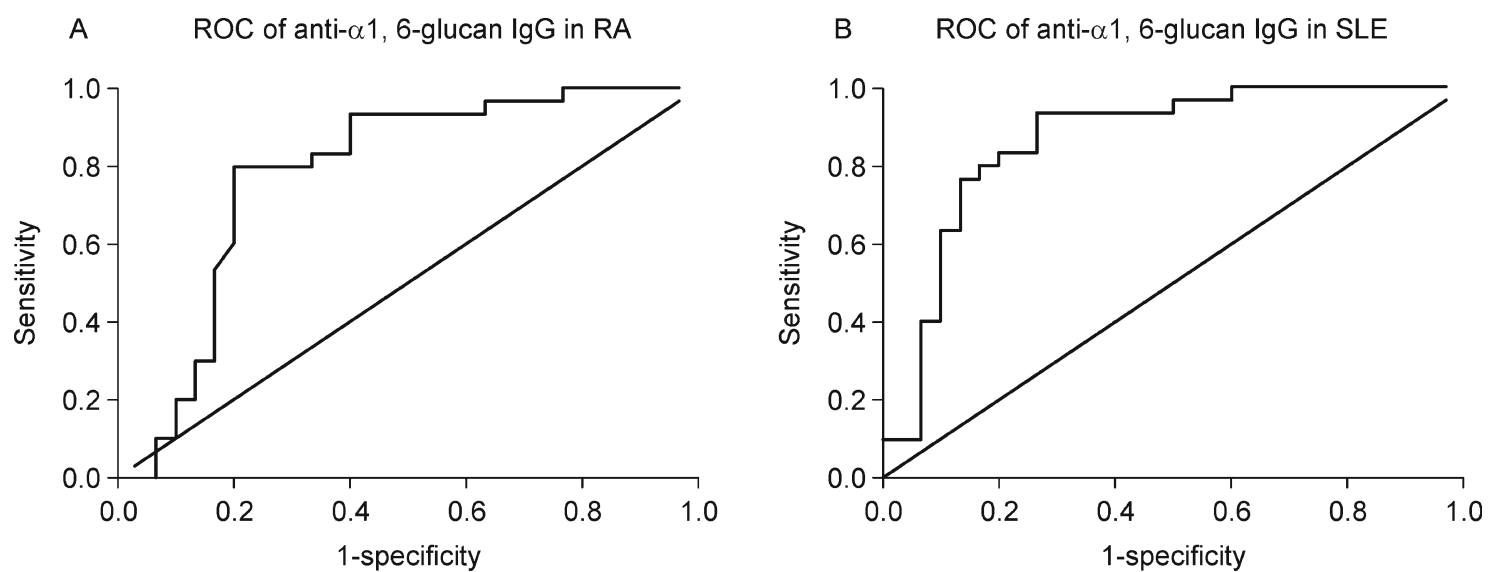

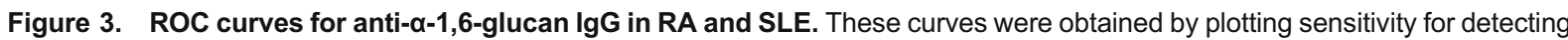
RA (A) or SLE (B) against specificity. For the anti- $\alpha-1,6-$ glucan IgG curve, healthy controls were used as the control group for determining specificity.

Table 3 Subject information

\begin{tabular}{lccc}
\hline Subject groups & NS & SLE & RA \\
\hline Number of subjects & 30 & 30 & 30 \\
Mean age, year (range) & $30.7(20-50)$ & $31.7(17-51)$ & $52.1(26-82)$ \\
Female/male & $16 / 14$ & $26 / 4$ & $21 / 9$ \\
Duration of disease, year (range) & - & $6.01(1-12)$ & $5.27(0.2-17)$ \\
\hline
\end{tabular}

levels strongly correlated with ANA $(r=0.675 ; p<0.05)$. Perhaps there is substantial cross-reactivity between dextran and dsDNA, and this notion would help to explain the fact that dextran can adsorb and remove anti-dsDNA antibody from sera of SLE patients. However, anti-dsDNA antibodies crossreact with many different antigens such as heparan sulfate, snRNP proteins, $\alpha$-actinin and nephritogenic potential (Mageed and Zack, 2002; Zhao et al., 2005). The crossreactivity with dextran is relatively low and should not affect the outcome of this study.

In conclusion, elevated levels of anti- $\alpha-1,6$-glucan serum antibodies correlate with the development of RA and SLE. These results provide not only valuable information on the mechanisms of interplay between glucan moieties and the human immune system, but also important clues for improved diagnosis and/or treatment of rheumatic diseases. The role of a-1,6-glucan-specific antibodies in immunological defense, immunoregulation and immunopathology merits further investigation.

\section{MATERIALS AND METHODS}

\section{Human subjects and blood samples}

Serum samples were collected from 30 healthy volunteers. An equal proportion mixture of sera from all 30 donors was prepared and employed as negative control sample. Blood samples were collected from a group of 30 hospitalized patients with RA, meeting the American College of Rheumatology 1987 Criteria for RA (Arnett et al., 1988). Their disease was in an active phase as defined by the criteria of the Chinese Association for Rheumatology; at the time of the blood collection their condition satisfied 4 of the 5 following criteria: (1) moderate resting pain; (2) morning stiffness $\geqslant 1 \mathrm{~h}$; (3) swelling of 3 or more joints; (4) $\geqslant 5$ tender joints; (5) erythrocyte sedimentation rate $(E S R) \geqslant 28 \mathrm{~mm} / \mathrm{h}$ (Westergren's method). Serum samples were also collected from 30 hospitalized patients with active SLE fulfilling the American Rheumatism Association Criteria for Diagnosis of SLE (Tan et al., 1982). All SLE patients donated blood samples while their disease was in flare. Five of them also donated while in a convalescence phase. Both RA and SLE patients attended the Department of Rheumatology and Immunology, Peking University People's Hospital, Beijing between 2006 and 2007. Patient characteristics are shown in Table 3. The blood samples were processed within $18 \mathrm{~h}$ of collection and the sera stored at $-80^{\circ} \mathrm{C}$ until use. The study has been reviewed and approved by the Ethics Committee of Peking University Health Science Center, Beijing, China.

\section{Polysaccharides-based ELISA}

Dextran ( $\alpha-1,6-$ glucan, MW 70,000, 17-0280-01) were purchased from Sigma (USA). Anti-a-1,6-glucan IgG, IgM and IgA antibodies were measured by ELISA. Flat bottom 96-well microtitre plates (Corning-Costar) were coated with $50 \mu \mathrm{g} / \mathrm{mL} \mathrm{\alpha -1,6-glucan} \mathrm{in} 0.1 \mathrm{~mol} /$ $\mathrm{L}$ carbonate/ bicarbonate buffer $(\mathrm{pH} 9.6)$ at $4^{\circ} \mathrm{C}$ overnight. The plates were washed with $0.05 \%$ Tween 20 (Sigma, St. Louis, Mo, USA) in 
PBS three times between each stage. Each plate was blocked with $10 \%$ FCS in PBS for $2 \mathrm{~h}$ at $37^{\circ} \mathrm{C}$. Serum samples were diluted $1: 200$ in $2 \%$ FCS in PBS and incubated in the ELISA wells for $2 \mathrm{~h}$ at $37^{\circ} \mathrm{C}$. Detection of $\operatorname{lgM}$, IgG or $\lg A$ was done using goat anti-human $\lg M$, IgG or IgA coupled to horseradish peroxidase (Southern Biotechnology Associates Inc., Birmingham, AL, USA) diluted 1:4000 in PBSTween and incubated for $1 \mathrm{~h}$ at $37^{\circ} \mathrm{C}$. The reaction was developed with $100 \mu \mathrm{L}$ of O-phenylenediamine (OPD, Sigma) for $5 \mathrm{~min}$ and stopped with $100 \mu \mathrm{L} 3 \mathrm{~mol} / \mathrm{L} \mathrm{H}_{2} \mathrm{SO}_{4}$. Optical density (OD) was measured at $492 \mathrm{~nm}$ using an ELISA spectrophotometer (Titertek Multiscan Plus MK II; ICN Flow Laboratories, Irvine, UK). The OD reading of the control sample on each plate, controlled at $0.2-0.3$ throughout the study, was used to calculate the binding index $(\mathrm{BI})$ of the specimen: binding index = OD (sample)/OD (control).

\section{Statistical analysis}

All serum samples were assayed for anti- $\alpha-1,6$-glucan-lgG, IgA and $\operatorname{lgM}$ at least 3 times with consistent results. Comparison of the groups was performed using the Spearman correlation analysis. Differences in the prevalence of the antibodies under study were analyzed by Fisher's Exact test in the disease groups and in controls. Significance was defined as a $p$ value of $<0.05$. A receiver operating characteristics (ROC) curve was generated by plotting sensitivity ( $y$ axis) against 1 -specificity (x axis). Statistical analysis was performed using SPSS software.

\section{ACKNOWLEDGEMENTS}

This study was supported by grants from the National Key Basic Research Program (Grant No. 2007CB512406), the National High Technology Research and Development Program of China (863 Program) (Grant No. 2006AA02Z495) and the National Natural Science Foundation of China (Grant No. 30371303).

\section{ABBREVIATIONS}

AHA, anti-histone antibody; AKA, antikeratin autoantibody; ANA, antinuclear antibody; APF, antiperinuclear factor; ARPA, anti-ribosomal $\mathrm{P}$ protein antibody; ASCMA, anti-Saccharomyces cerevisiae mannan antibody; CCP, anti-cyclic citrullinated peptide; CRP, Creactive protein; ESR, erythrocyte sedimentation rate; HRFIgA /lgG, hidden rheumatoid factor for $\lg A / \operatorname{lgG}$; RA, rheumatoid arthritis; ROC, receiver operating characteristics; RF, rheumatoid factor; SLE, systemic lupus erythematosus; $\mathrm{Sm}$, anti-Smith antibody

\section{REFERENCES}

Anastase, S., Letourneur, D., and Jozefonvicz, J. (1996). Affinity chromatography of human anti-dextran antibodies. Isolation of two distinct populations. J Chromatogr B Biomed Appl 686, 141-150.

Arnett, F.C., Edworthy, S.M., Bloch, D.A., McShane, D.J., Fries, J.F., Cooper, N.S., Healey, L.A., Kaplan, S.R., Liang, M.H., Luthra, H. S., et al. (1988). The American Rheumatism Association 1987 revised criteria for the classification of rheumatoid arthritis. Arthritis Rheum 31, 315-324.

Asahi, T., Yamamoto, T., and Kutsuki, H. (2003). Blood purification therapies using dextran sulfate cellulose columns Liposorber and
Selesorb. Ther Apher Dial 7, 73-77.

Bourne, J.T., Kumar, P., Huskisson, E.C., Mageed, R., Unsworth, D. J., and Wojtulewski, J.A. (1985). Arthritis and coeliac disease. Ann Rheum Dis 44, 592-598.

Braun, N., Jünger, M., Klein, R., Gutenberger, S., Guagnin, M., and Risler, T. (2002). Dextran sulfate (Selesorb) plasma apheresis improves vascular changes in systemic lupus erythematosus. Ther Apher 6, 471-477.

Chacko, B.K., and Appukuttan, P.S. (2003). Dextran-binding human plasma antibody recognizes bacterial and yeast antigens and is inhibited by glucose concentrations reached in diabetic sera. Mol Immunol 39, 933-939.

Dai, H., Li, Z., Zhang, Y., Lv, P., and Gao, X.M. (2009a). Elevated levels of serum antibodies against Saccharomyces cerevisiae mannan in patients with systemic lupus erythematosus. Lupus 18 , 1087-1090.

Dai, H., Li, Z., Zhang, Y., Lv, P., and Gao, X.M. (2009b). Elevated levels of serum IgA against Saccharomyces cerevisiae mannan in patients with rheumatoid arthritis. Cell Mol Immunol 6, 361-366.

Dinglasan, R.R., Valenzuela, J.G., and Azad, A.F. (2005). Sugar epitopes as potential universal disease transmission blocking targets. Insect Biochem Mol Biol 35, 1-10.

Galili, U. (2001). The alpha-gal epitope (Gal alpha 1-3Gal beta 14GIcNAc-R) in xenotransplantation. Biochimie 83, 557-563.

Galili, U. (2005). The alpha-gal epitope and the anti-Gal antibody in xenotransplantation and in cancer immunotherapy. Immunol Cell Biol 83, 674-686.

Greenstein, A.J., Janowitz, H.D., and Sachar, D.B. (1976). The extraintestinal complications of Crohn's disease and ulcerative colitis: a study of 700 patients. Medicine (Baltimore) 55, 401-412.

Gupta, D., Kaltner, H., Dong, X., Gabius, H.J., and Brewer, C.F. (1996). Comparative cross-linking activities of lactose-specific plant and animal lectins and a natural lactose-binding immunoglobulin $\mathrm{G}$ fraction from human serum with asialofetuin. Glycobiology 6, 843-849.

Hashimoto, H., Tsuda, H., Kanai, Y., Kobayashi, S., Hirose, S., Shinoura, H., Yokohari, R., Kinoshita, M., Aotsuka, S., Yamada, H., et al. (1991). Selective removal of anti-DNA and anticardiolipin antibodies by adsorbent plasmapheresis using dextran sulfate columns in patients with systemic lupus erythematosus. J Rheumatol 18, 545-551.

Huang, C.Y., Thayer, D.A., Chang, A.Y., Best, M.D., Hoffmann, J., Head, S., and Wong, C.H. (2006). Carbohydrate microarray for profiling the antibodies interacting with Globo $\mathrm{H}$ tumor antigen. Proc Natl Acad Sci U S A 103, 15-20.

Kutsuki, H., Takata, S., Yamamoto, K., and Tani, N. (1998). Therapeutic selective adsorption of anti-DNA antibody using dextran sulfate cellulose column (Selesorb) for the treatment of systemic lupus erythematosus. Ther Apher 2, 18-24.

Mageed, R.A., and Zack, D.J. (2002). Cross-reactivity and pathogenicity of anti-DNA autoantibodies in systemic lupus erythematosus. Lupus 11, 783-786.

Matsuki, Y., Suzuki, K., Kawakami, M., Ishizuka, T., Kawaguchi, Y., Hidaka, T., and Nakamura, H. (1996). High-avidity anti-DNA antibody removal from the serum of systemic lupus erythematosus patients by adsorption using dextran sulfate cellulose columns. J Clin Apher 11, 30-35. 
Pal, S., Bandyopadhyay, S., Chatterjee, M., Bhattacharya, D.K., Minto, L., Hall, A.G., and Mandal, C. (2004). Antibodies against 9O-acetylated sialoglycans: a potent marker to monitor clinical status in childhood acute lymphoblastic leukemia. Clin Biochem 37, 395- 403.

Suzuki, K. (2000). The role of immunoadsorption using dextransulfate cellulose columns in the treatment of systemic lupus erythematosus. Ther Apher 4, 239-243.
Tan, E.M., Cohen, A.S., Fries, J.F., Masi, A.T., McShane, D.J., Rothfield, N.F., Schaller, J.G., Talal, N., and Winchester, R.J. (1982). The 1982 revised criteria for the classification of systemic lupus erythematosus. Arthritis Rheum 25, 1271-1277.

Zhao, Z., Weinstein, E., Tuzova, M., Davidson, A., Mundel, P., Marambio, P., and Putterman, C. (2005). Cross-reactivity of human lupus anti-DNA antibodies with alpha-actinin and nephritogenic potential. Arthritis Rheum 52, 522-530. 